\title{
Innervation of a Prefabricated Flap: A New Experimental Model
}

\author{
Marco Romeo, ${ }^{1}$ Giuseppe Cuccia, ${ }^{2}$ Shan Shan Qiu, ${ }^{1}$ Stefania Raimondo, ${ }^{3}$ \\ Stefano Geuna, ${ }^{3}$ and Bernardo Hontanilla ${ }^{1}$ \\ ${ }^{1}$ University Clinic of Navarra, Plastic Surgery Unit, Avenida Pio XII 36, 30001 Pamplona, Spain \\ ${ }^{2}$ Fatebenefratelli Buccheri La Ferla Hospital, Plastic Surgery Unit, 90123 Palermo, Italy \\ ${ }^{3}$ Department of Clinical and Biological Sciences, University of Turin, Regione Gonzole 10, Orbassano, 10043 Turin, Italy
}

Correspondence should be addressed to Marco Romeo; romeoplastica@gmail.com

Received 4 March 2014; Accepted 30 June 2014; Published 24 July 2014

Academic Editor: Ulrich Kneser

Copyright (C) 2014 Marco Romeo et al. This is an open access article distributed under the Creative Commons Attribution License, which permits unrestricted use, distribution, and reproduction in any medium, provided the original work is properly cited.

\begin{abstract}
Introduction. Flap innervation by neoaxonogenesis is a promising field of investigation. The authors evaluated the possibility of innervating an acellular collagen scaffold as component of a potential prefabricated flap. Materials and Methods. Collagen matrix sheets were implanted around the femoral bundle of a murine model to produce two flaps on proximal and distal nerve stumps based on a flow-through model. After thirty days, nerve regeneration and integration into the collagen matrix were evaluated. The specimens were microscopically analyzed to study Schwann cell colonization and axonal integration with the matrix. Axonal count and density were assessed and statistically evaluated. Results. Qualitative structural and ultrastructural evaluation indicated integration, with axonal fibers merged within the collagen matrix, along with a newly formed vascular network on the proximal flap. Wallerian degeneration occurred inside the distal chamber. Axonal count and density did not show statistically significant differences between the nerve inside the proximal flap and the control side. Conclusions. Innervation of an acellular matrix can be obtained by direct nerve stump implantation. The flow-through system was relatively easy to build and reliable to provide adequate blood supply. The collagen scaffold may be a promising support or further studies of preinnervated microsurgical flaps.
\end{abstract}

\section{Introduction}

Tissue engineering is raising great expectations in regenerative medicine. It aims to understand the basic mechanism of tissue growth to bridge from an ex vivo to an in vivo setting aimed at facilitating clinical applications [1] to repair, maintain, and eventually improve tissue/organ function.

Bitto et al. [2] and Messina et al. [3] investigated the integration of a nerve stump in a AV-loop prefabricated flap as source of stimulation for target tissues (fat or muscle). In this study we explored the possibility of performing innervation of an acellular dermal matrix (using the in vivo flow-through model tested by Tanaka et al. [4]) by creating a sealed vascularized flap including a peripheral nerve.

\section{Material and Methods}

Sixteen male Wistar rats (average weight 300 gr.) were operated at CIFA (Centre for Applied Pharmacological Investigation, University of Navarra, Pamplona, Spain); power analysis was performed to ensure statistic consistence of the samples. Experiments, care, and treatment of the animals were consistent with national and EU laws and policies (EEC Council Directive 86/609, OJL 358, December 12, 1987). All operations were carried out under general anaesthesia using an intraperitoneal injection of Ketamine $(75 \mathrm{mg} / \mathrm{kg})$ and Xylazina $(10 \mathrm{mg} / \mathrm{kg})$; each animal was kept in a single cage and euthanized at the end of observation period with an overdose of anesthesia.

2.1. Flap Construction. Femoral bundles were exposed on both limbs. The right thigh nerve was dissected from artery and vein, transected, and used as a control group for normal femoral nerve morphology at day zero and after one month (Figure 1).

On the left limb the artery and vein were kept intact and freely dissected from the collateral vessels to maintain the flow-through model. The nerve was cut leaving a $20 \mathrm{~mm}$ gap between the two ends and the collagen scaffold sheets 


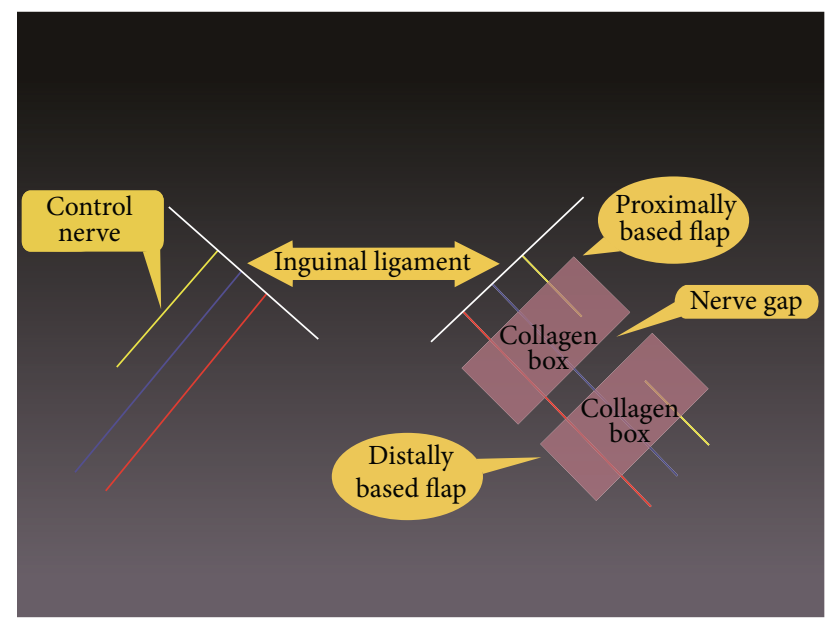

FIgURE 1: Schematic representation of study concept and flaps construction. On the right limb (left on the image) the femoral nerve was cut and left to physiological growth evolution. On the left limb (right on the image) the femoral nerve was cut and the proximal and distal stumps were buried in the collagen sheets (pink rectangles) and sealed with the silicon layer. The vascular bundle (blue and red lines) was freely dissected from collateral vessels to maintain the flow-through model.

(Integra, Plainsboro, New Jersey, USA) were folded around the bundle to create two $15 \times 15 \mathrm{~mm}$ flaps. The nerve was stitched to the collagen matrix with $9 / 0$ nylon to prevent accidental extrusion; a $15 \mathrm{~mm}$ gap was left between the flaps. The outer silicon layer was left to isolate the flaps from external contaminations and closed with running $6 / 0$ Ethilon. A further stich was placed to anchor each flap to surrounding tissues to avoid accidental malposition, rotation, flaps contact, and torsion of the pedicle (Figure 2).

2.2. Histological and Biological Analysis. At day zero, the control nerve was cut for morphological evaluation.

After thirty days, the patency test was performed by clamping the artery, emptying a tract of the vessel and reestablishing the flow, the same was done with the vein to confirm adequate outflow, and the flaps were then removed. All sixteen rats had a $2 \mathrm{~mm}$ portion of the stump of the control nerve cut and studied.

The rats were randomly divided in two groups to study the flaps in the left thigh: in eight rats, the outer silicone layer only was removed and whole collagen-vessels-nerve complex was embedded in paraffin and processed for histological and immunohistochemical analysis. Specimens were stained with Masson trichrome method (Bio-Optica, Milan, Italy). For immunohistochemistry, the following antibodies were used: anti-S100 (polyclonal, rabbit, dilution 1: 800, Sigma, St. Louis, $\mathrm{MO}$ ), for detecting Schwann cells, and anti-beta-tubulin (monoclonal, mouse, dilution 1:1,000, Sigma, St. Louis, MO), for detecting axons. In the other eight rats, both flaps were opened and the nerve stump was carefully dissected and harvested, embedded in resin, and processed for high-resolution light and electron microscopy and histomorphometry. For light microscopy and histomorphometry, semithin sections
$(2.5 \mu \mathrm{m})$ were stained by toluidine blue and examined in a DM4000B microscope equipped with a DFC320 digital camera (Leica Microsystems, Wetzlar, Germany).

For electron microscopy, ultrathin sections $(70 \mathrm{~nm})$ were stained with uranyl acetate and lead citrate and examined in a JEM-1010 transmission electron microscope (JEOL, Tokyo, Japan). Axonal count was performed with Image (National Institute of Health, USA) by analyzing standardized images of sections of the nerves at $300 \mathrm{dpi}$ of resolution. Axonal density was performed by simple pixel/mm conversion delimitating an area in the core of the nerve of known size [5].

All data collected from axonal count/density groups were analyzed with a Pearson test to verify correlation between overall number of axons per section and density before and after the observation period. The Fischer test was used to observe any significant difference among the proximal, distal, and control groups evaluating the number and density of axons $/ \mathrm{mm}^{2}$.

\section{Results}

No rats died and no complications were encountered apart from a silicon layer extrusion. The authors avoided this problem using the inguinal fat pad to bury the flap. At day thirty, before removing the flaps, patency test was $100 \%$ positive for distal perfusion and no vascular failure was recorded.

3.1. Microscopy and Immunohistochemistry Analysis. In both proximal and distal flaps, trichrome staining has been performed and images at different magnifications are presented in Figure 3. Low magnifications (Figures 3(a) and 3(b)) allowed visualizing the entire flap and suggested the formation of more abundant tissue with a richer vascular network in the proximal flap (Figure 3(a)) than in the distal one (Figure 3(b)). The collagen matrix (blue stained) had been colonized by abundant new tissue especially in the proximal flap. Within the neurovascular bundle, the femoral nerve (arrows in Figures 3(a)-3(f)) can be observed close to the vessels.

Higher magnification of femoral nerve showed a different morphology between proximal (Figure 3(g)) and distal (Figure 3(h)) flap. Axons (red stained) were more abundant in the proximal part than in the distal. Immunohistochemistry after axonal and glial marker employment revealed that, in the proximal stump, both axons (green) and Schwann cells (red) were still present (box in Figure 3(g)). By contrast, in the distal stump only glial cell markers, but not axonal markers, were detected (box in Figure 3(h)). This indicated the occurrence of selective axonal involution typical of distal Wallerian degeneration. Moreover in the proximal flap, 30 days after surgery several sprouted fibers are detected in the collagen matrix around the neurovascular bundle (Figures $3(\mathrm{i})-3(\mathrm{k}))$.

High resolution light and electron microscopy (Figure 4) confirmed that the proximal nerve stump (Figures 4(c) and $4(\mathrm{~d})$ ) was organized similarly to the control nerve (Figures 4(a) and 4(b)). Wallerian degeneration occurred in 


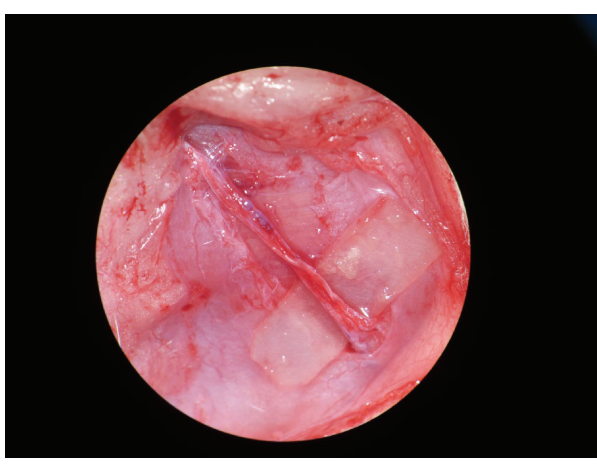

(a)

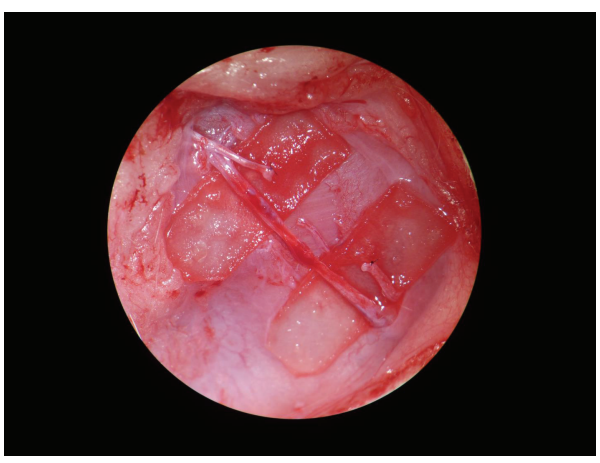

(c)

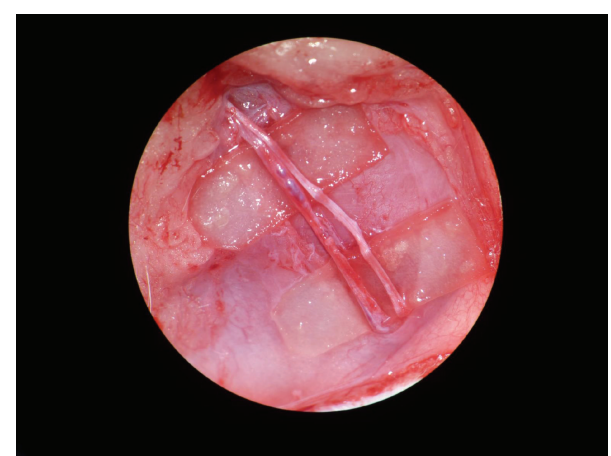

(b)

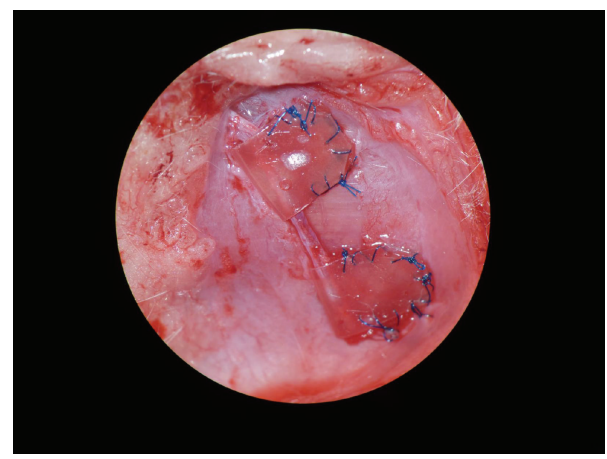

(d)

Figure 2: Construction phases of the two flaps. (a) The pedicle is harvested, the nerve separated, and the first piece of Integra is placed. (b) The two layers of collagen are placed. (c) The nerve is cut and the two stumps are secured onto the collagen. (d) The boxes are sealed with nylon suture and anchored to the underlying muscle.

the distally based stump (Figures 4(e) and 4(f)), where abundant myelin destruction can be observed.

Proximal flap's wall images with electron microscopy showed the presence of random nerve fibers and vessels merged into the collagen matrix (Figure 5).

3.2. Nerve Count and Density. Histomorphometry (number of myelinated fibers and axonal density) has been performed on control and proximal and distal femoral nerve immediately (Day 0 ) and 30 days (Day 30) after surgery as reported in Table 1. No significant $(P>0.05)$ differences have been observed between control and proximal femoral nerve, both at day 0 and day 30 . The distal stump at day 30 showed no countable amount of axons.

Pearson's test gave a weak $(r=0.42)$ correlation between average axons number and density of axon $/ \mathrm{mm}^{2}$ for the control nerve and a mild positive correlation for the proximally based nerve $(r=0.66)$.

\section{Discussion}

4.1. Vascular Preconditioning. An adequate vascular support has been shown to be a basic condition to promote nerve outgrowth in the matrix. Neoangiogenesis is a time related process; therefore it is important to allow sufficient time to develop a new vascular network and nerve regeneration with it.
According to Pribaz and Fine [6], a prefabricated flap requires an 8-week maturation of a vascular pedicle inside a volume of tissue to promote neovascularization. Although this definition is still widely accepted, other authors have published similar results with shorter time of neovascularization. Muller et al. [7] achieved vascularization of a synthetic resorbable scaffold based on the epigastric bundle of rat within three weeks, while Wang et al. [8] demonstrated angiogenesis after just two weeks. MacLeod et al. [9] found vascularity on a dermal matrix after two weeks on a rat model based on the epigastric artery (similar to our model) and successfully implanted it as a microvascular free flap. Houle and Neumeister brought these principles into the clinical setting, creating a completely new free flap [10].

According to Tanaka et al. [4] the flow-through model is a simpler but equally reliable system to A-V microsurgical loop flap [11] and might represent an adequate model for further studies. The good sealing achieved with the outer silicon layer and subsequent capsular formation guarantee a sufficiently isolated scenario without external influences, that might be ideal for testing growth factors within the flap as bioreactor.

4.2. Matrix Innervation. Other authors have previously investigated the possibility of innervation of an artificial construct with different aim or kind of scaffold. Suuronen et al. [12] were able to innervate a tissue-engineered cornea adding neural growth factor to promote SC (Schwann cells) 


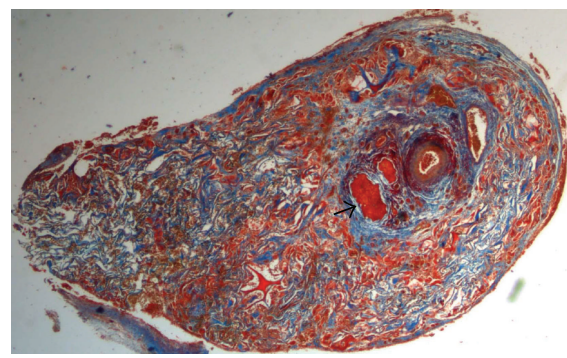

(a)

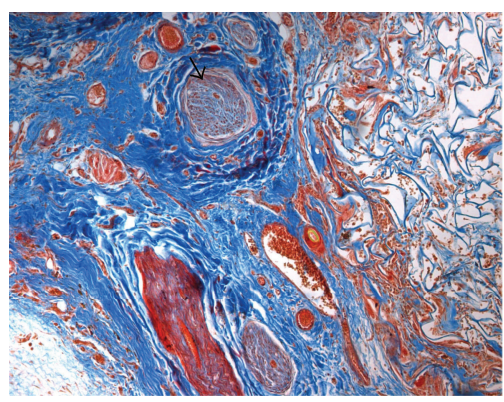

(d)

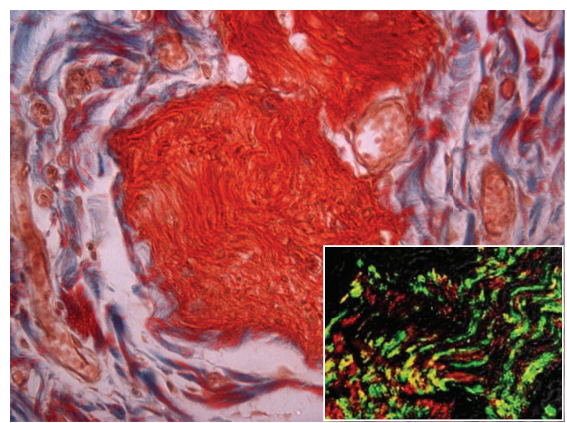

(g)

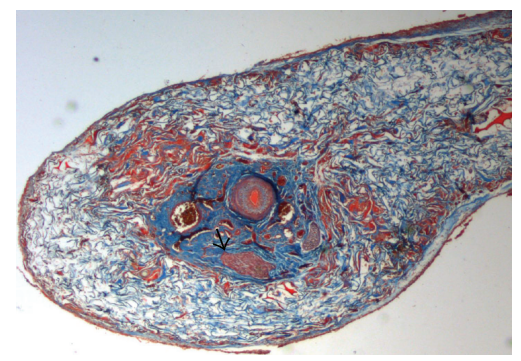

(b)

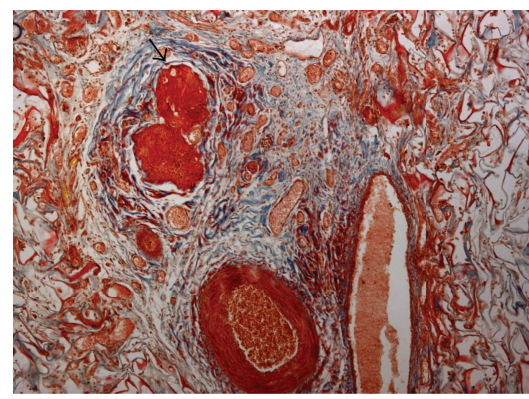

(e)

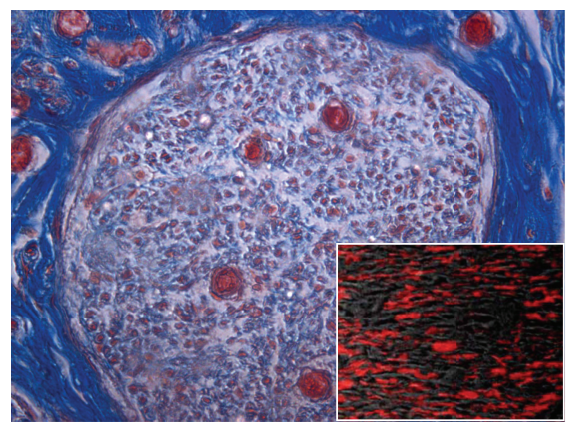

(h)

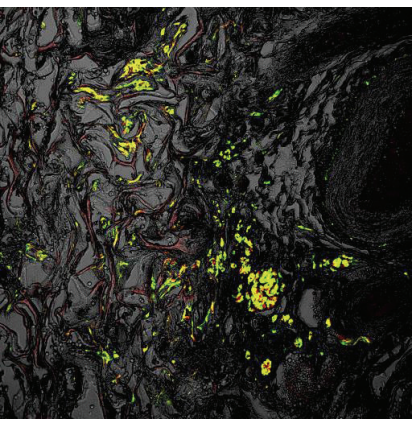

(k)

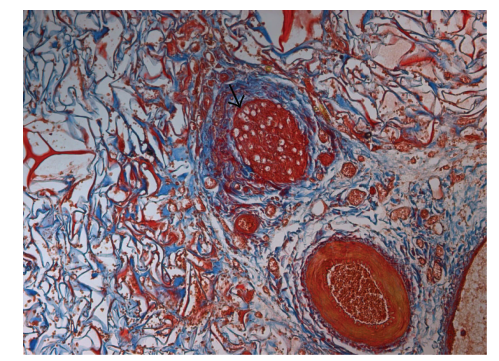

(c)

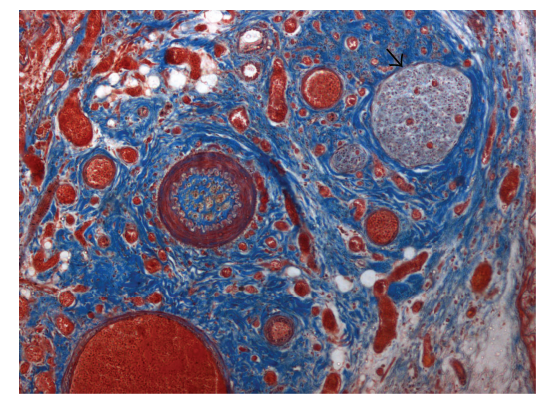

(f)

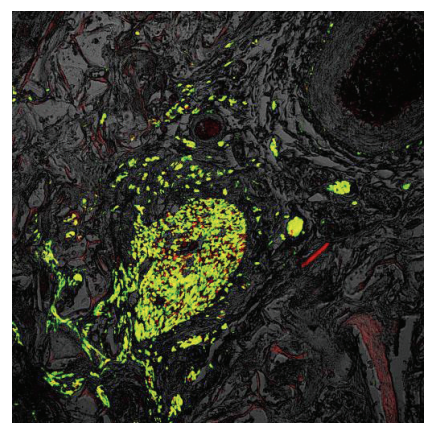

(i)

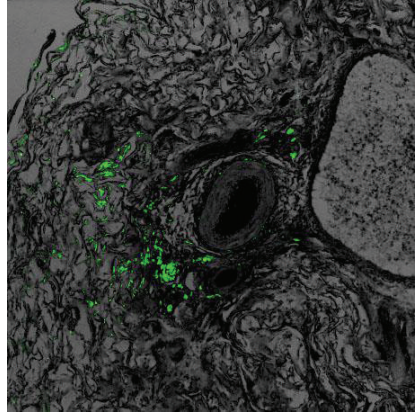

(j)

FiguRE 3: Light microscopy (trichrome staining) and immunofluorescence (S-100-red and beta-tubulin-green) of paraffin sections from collagen chambers with proximal nerve stump ((a), (c), (e), (g), (i), (j), and (k)) and distal nerve stump ((b), (d), (f), and (h)). The arrows point to the nerve located close to the vascular bundle. Original magnification: (a), (b) =25x; (c), (d) = 100x; (e), (f) = 200x; (g), (h) = 400x; box in $(\mathrm{g})$ and $(\mathrm{h})=600 \mathrm{x} ;(\mathrm{i}),(\mathrm{k})=100 \mathrm{x} ;(\mathrm{j})=80 \mathrm{x}$.

progression. Parenteau-Bareil et al. [13] affirmed that a threedimensional tissue-engineered nervous system model can be used to promote axonal migration by SC stimulation through a connective tissue (due to its porous and reabsorbable characteristics). Fukushima et al. [14] successfully tested a honeycomb collagen scaffold to reinnervate a transected spinal cord.

Similar to neoangiogenesis, innervation of artificial flaps can either follow an extrinsic pathway or ensue from intrinsic innervation. In the former condition, the axonal colonization 


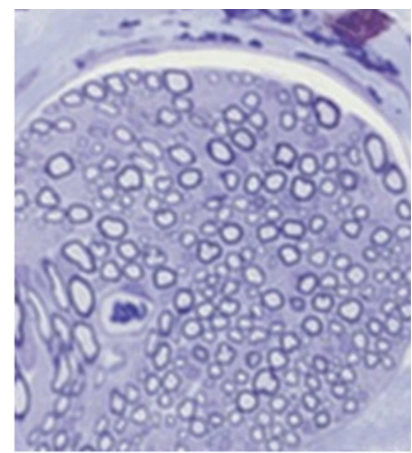

(a)

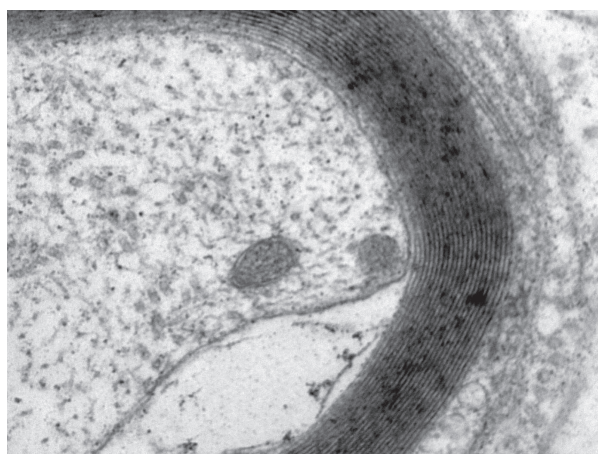

(d)

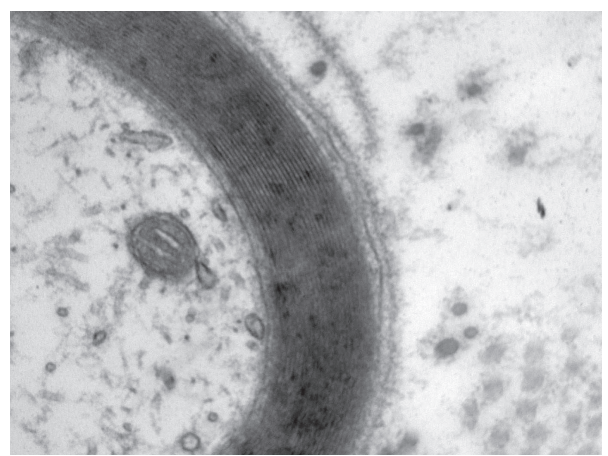

(b)

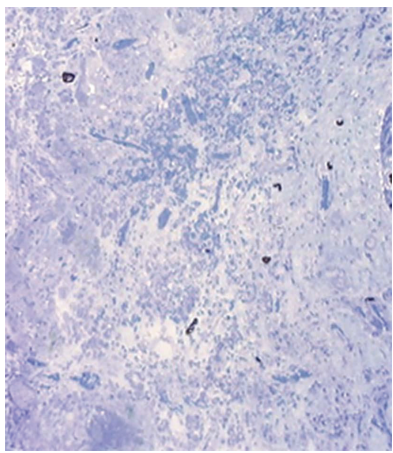

(e)

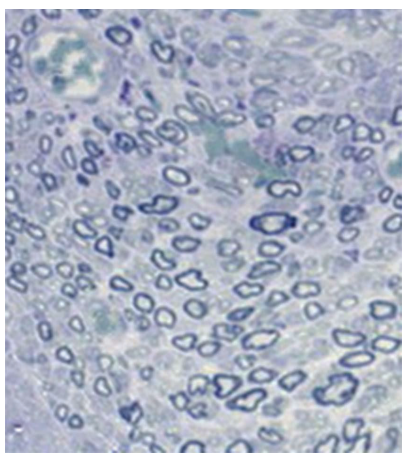

(c)

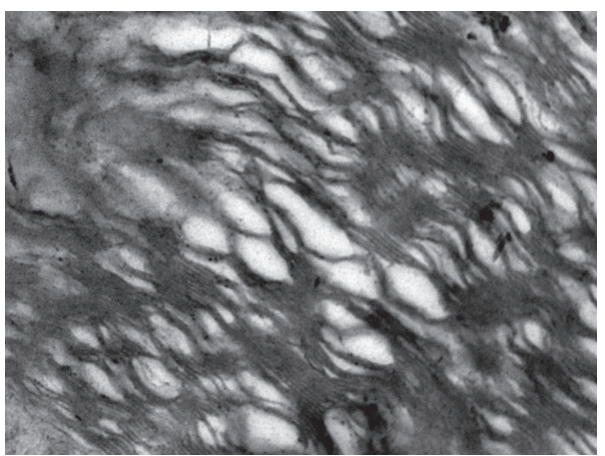

(f)

FIGURE 4: High resolution light microscopy ((a), (c), and (e)) and electron microscopy ((b), (d), and (f)) of control femoral nerve ((a), (b)), proximal nerve stump ((c), (d)), and distal nerve stump ((e), (f)) buried in the artificial flap. Original magnification: (a), (c), and (e) = 400x; (b), (d), and (f) $=80,000 x$.

TABLE 1: Histomorphometrical evaluation of myelinated fibers and axonal density in control and proximal femoral nerve.

\begin{tabular}{|c|c|c|c|c|}
\hline & \multicolumn{2}{|c|}{ Day 0} & \multicolumn{2}{|c|}{ Day 30} \\
\hline & Number of fibers & Axonal density & Number of fibers & Axonal density \\
\hline \multicolumn{5}{|l|}{ Control nerve } \\
\hline Mean \pm SEM & $1467 \pm 203$ & $8596 \pm 253$ & $1663 \pm 229$ & $8215 \pm 305$ \\
\hline \multicolumn{5}{|l|}{ Proximal nerve } \\
\hline Mean \pm SEM & $1564 \pm 158$ & $8639 \pm 258$ & $1700 \pm 152$ & $8883 \pm 259$ \\
\hline
\end{tabular}

of the flap is limited by the absence of SC that guide axons and support formation of functionally active nerve fibers. Schwann cells express surface proteins and elaborate growth factors that lead to the extension of the regenerating axonal ends $[15,16]$.

We hypothesized that, to overcome this limitation, intrinsic preinnervation and flap vascular preconditioning should be sought. We tested preinnervation by using proximal and distal nerve stumps. As expected Wallerian degeneration of the axons occurred in the distally based flap [17].

On the other hand, the proximal construct showed nerve integration within the collagen matrix. Immunohistochemistry (Figure 3) and electron microscopy (Figure 5) demonstrated that from the main stump several fibers invaded the collagen flap with a vascular sprout inside the collagen matrix next to it. An ordinate pattern of Schwann cells and the absence of scarring exclude the possibility of confusing this pattern with a newly formed neuroma [18-20].

\section{Conclusion}

Various prefabricated flaps have been described in the past. Preinnervated vascularized flaps represent a promising new class of bioengineered in vivo constructs that might improve the effectiveness of advanced tissue engineering and organ regeneration.

\section{Conflict of Interests}

The authors declare that there is no conflict of interests regarding the publication of this paper.

\section{Acknowledgments}

Marco Romeo and Giuseppe Cuccia share the same merit as the first author. This paper has been presented to the ECSAPS 


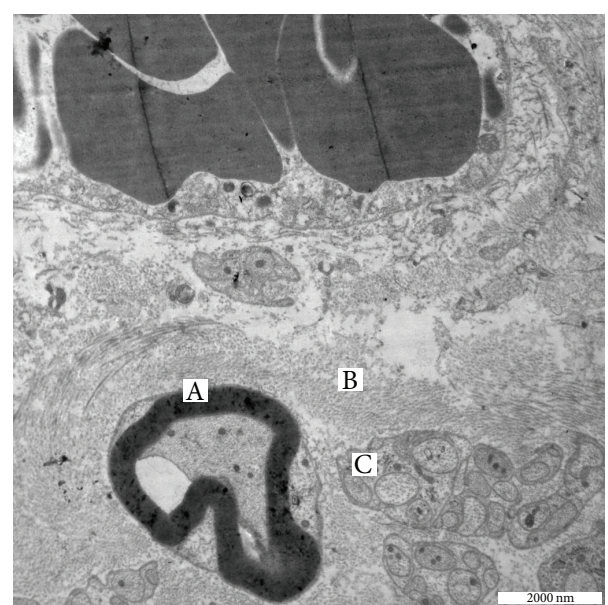

Figure 5: Electron microscopy image of the peripheral collagen layer. (A) Newly formed myelinated fibers. (B) Collagen fibres. (C) Nerve sprouting of unmyelinated fibers. A vessel can be seen on the top center of the image.

meeting in Pamplona, Spain, 28-29 October 2011, and SIM meeting, Palermo, Italy, 20-22 October 2011.

\section{References}

[1] B. D. MacArthur and R. O. C. Oreffo, "Bridging the gap," Nature, vol. 433, no. 7021, p. 19, 2005.

[2] F. F. Bitto, D. Klumpp, C. Lange et al., "Myogenic differentiation of mesenchymal stem cells in a newly developed neurotised AVloop model," BioMed Research International, vol. 2013, Article ID 935046, 11 pages, 2013.

[3] A. Messina, S. K. Bortolotto, O. C. S. Cassell, J. Kelly, K. M. Abberton, and W. A. Morrison, "Generation of a vascularized organoid using skeletal muscle as the inductive source," The FASEB Journal, vol. 19, no. 11, pp. 1570-1572, 2005.

[4] Y. Tanaka, K. Sung, A. Tsutsumi, S. Ohba, K. Ueda, and W. A. Morrison, "Tissue engineering skin flaps: which vascular carrier, arteriovenous shunt loop or arteriovenous bundle, has more potential for angiogenesis and tissue generation?" Plastic and Reconstructive Surgery, vol. 112, no. 6, pp. 1636-1644, 2003.

[5] M. H. Grider, Q. Chen, and H. D. Shine, "Semi-automated quantification of axonal densities in labeled CNS tissue," Journal of Neuroscience Methods, vol. 155, no. 2, pp. 172-179, 2006.

[6] J. J. Pribaz and N. A. Fine, "Prelamination: defining the prefabricated flap-a case report and review," Microsurgery, vol. 15, no. 9, pp. 618-623, 1994.

[7] D. Muller, H. Chim, A. Bader, M. Whiteman, and J.-T. Schantz, "Vascular guidance: microstructural scaffold patterning for inductive neovascularization," Stem Cells International, vol. 2011, Article ID 547247, 6 pages, 2011.

[8] H. Wang, X. Chang, G. Qiu et al., "Axial vascularization of nano-HA/collagen/PLA composites by arteriovenous bundle," Journal of Nanomaterials, vol. 2013, Article ID 391832, 6 pages, 2013.

[9] T. M. MacLeod, G. Williams, R. Sanders, and C. J. Green, "Prefabricated skin flaps in a rat model based on a dermal replacement matrix PermacolŮ," British Journal of Plastic Surgery, vol. 56, no. 8, pp. 775-783, 2003.
[10] J. M. Houle and M. W. Neumeister, "A prefabricated, tissueengineered integra free flap," Plastic and Reconstructive Surgery, vol. 120, no. 5, pp. 1322-1325, 2007.

[11] B. Manasseri, G. Cuccia, S. Moimas et al., "Microsurgical arterovenous loops and biological templates: a novel in vivo chamber for tissue engineering," Microsurgery, vol. 27, no. 7, pp. 623-629, 2007.

[12] E. J. Suuronen, M. Nakamura, M. A. Watsky et al., "Innervated human corneal equivalents as in vitro models for nerve-target cell interactions," The FASEB Journal, vol. 18, no. 1, pp. 170-172, 2004.

[13] R. Parenteau-Bareil, R. Gauvin, and F. Berthod, "Collagenbased biomaterials for tissue engineering applications," Materials, vol. 3, no. 3, pp. 1863-1887, 2010.

[14] K. Fukushima, M. Enomoto, S. Tomizawa et al., "The axonal regeneration across a honeycomb collagen sponge applied to the transected spinal cord," Journal of Medical and Dental Sciences, vol. 55, no. 1, pp. 71-79, 2008.

[15] S. Hall, "Axonal regeneration through acellular muscle grafts," Journal of Anatomy, vol. 190, part 1, pp. 57-71, 1997.

[16] A. Baron-Van Evercooren, H. K. Kleinman, S. Ohno, P. Marangos, J. P. Schwartz, and M. E. Dubois-Dalcq, "Nerve growth factor, laminin, and fibronectin promote neurite growth in human fetal sensory ganglia cultures," Journal of Neuroscience Research, vol. 8, no. 2-3, pp. 179-193, 1982.

[17] F. Buchthal and V. Kühl, "Nerve conduction, tactile sensibility, and the electromyogram after suture or compression of peripheral nerve: a longitudinal study in man," Journal of Neurology Neurosurgery and Psychiatry, vol. 42, no. 5, pp. 436-451, 1979.

[18] I. Ayan, A. Bora, M. Karakaplan et al., "Effect of end-to-side repair of proximal nerve stumps of transected peripheral nerves on the development of neuroma (experimental study)," Hand, vol. 2, no. 4, pp. 199-205, 2007.

[19] T. R. Tyner, N. Parks, S. Faria et al., "Effects of collagen nerve guide on neuroma formation and neuropathic pain in a rat model," The American Journal of Surgery, vol. 193, no. 1, pp. e1e6, 2007.

[20] S. Geuna, S. Raimondo, G. Ronchi et al., "Chapter 3 histology of the peripheral nerve and changes occurring during nerve regeneration," International Review of Neurobiology, vol. 87, pp. 27-46, 2009. 

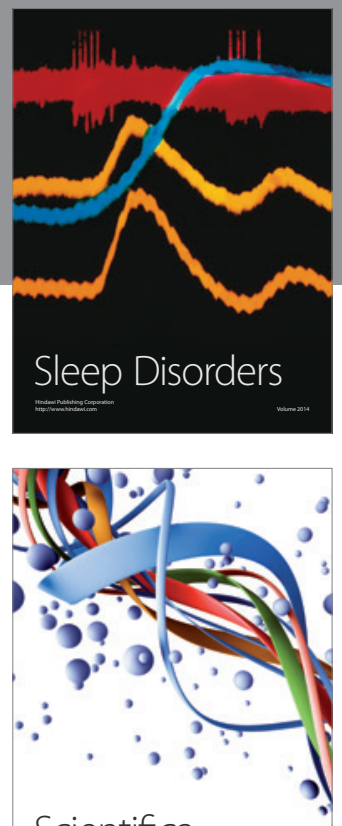

Scientifica
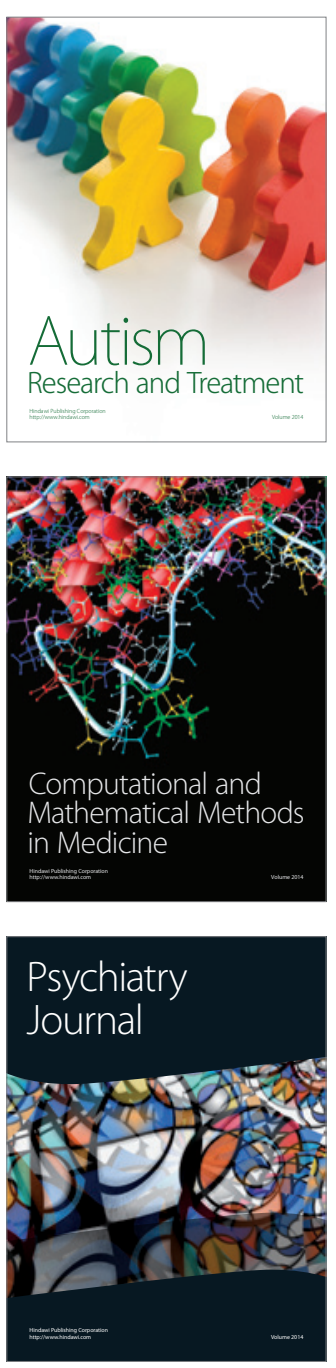
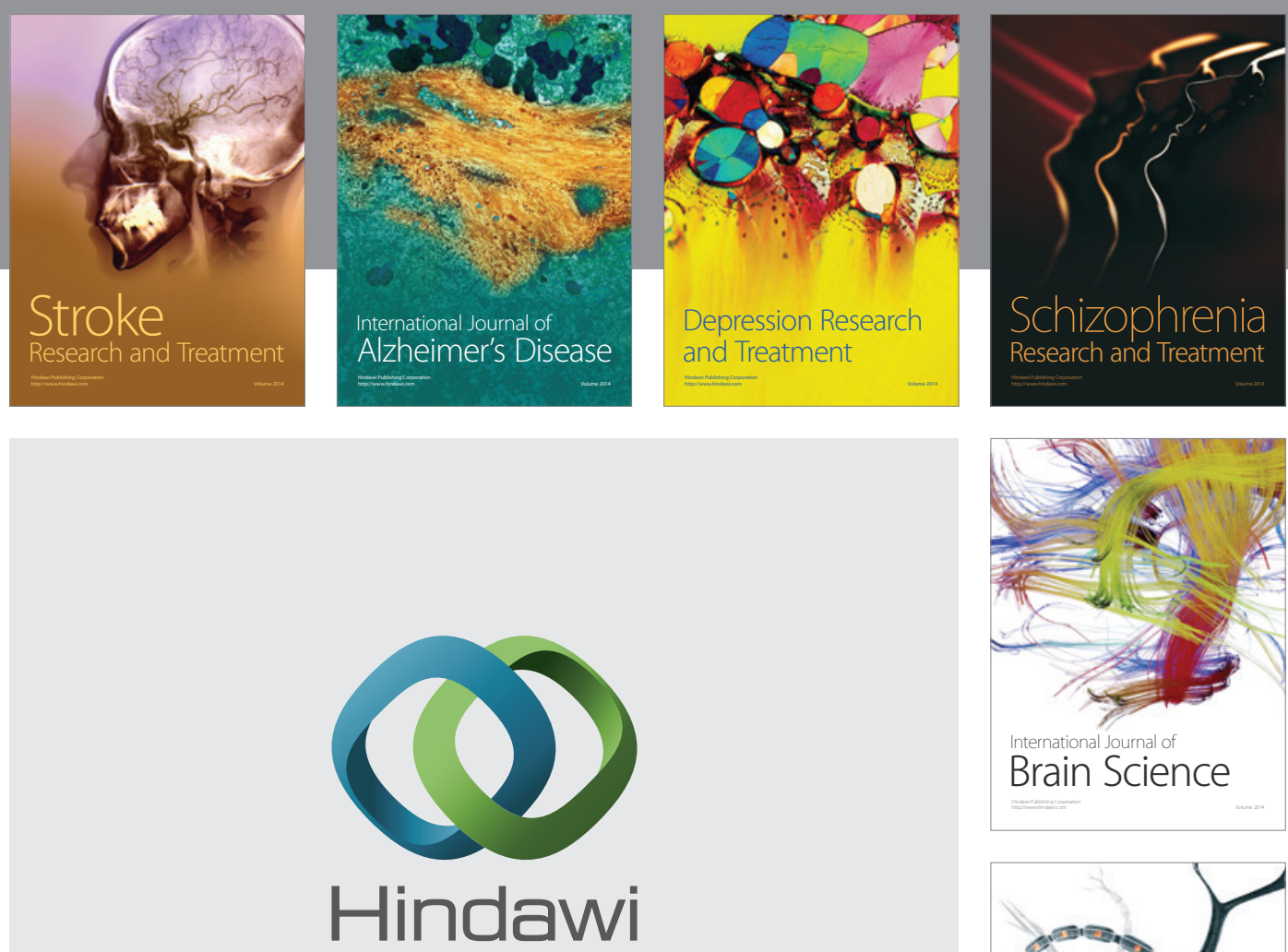

Submit your manuscripts at

http://www.hindawi.com
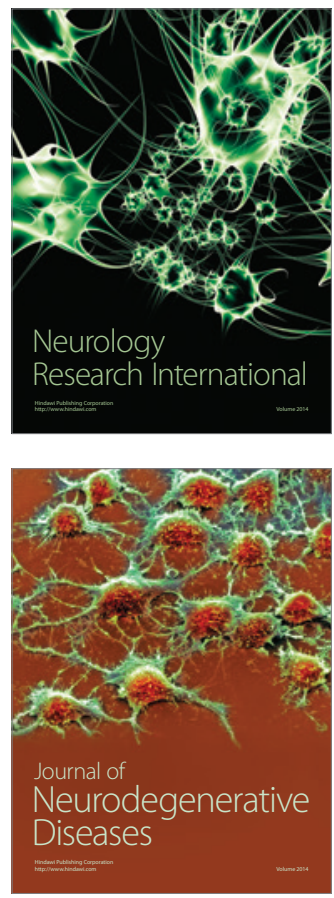

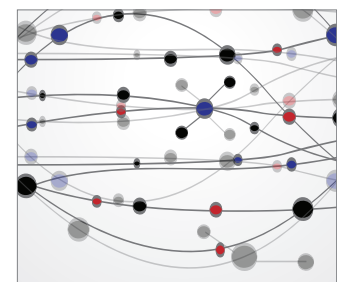

The Scientific World Journal
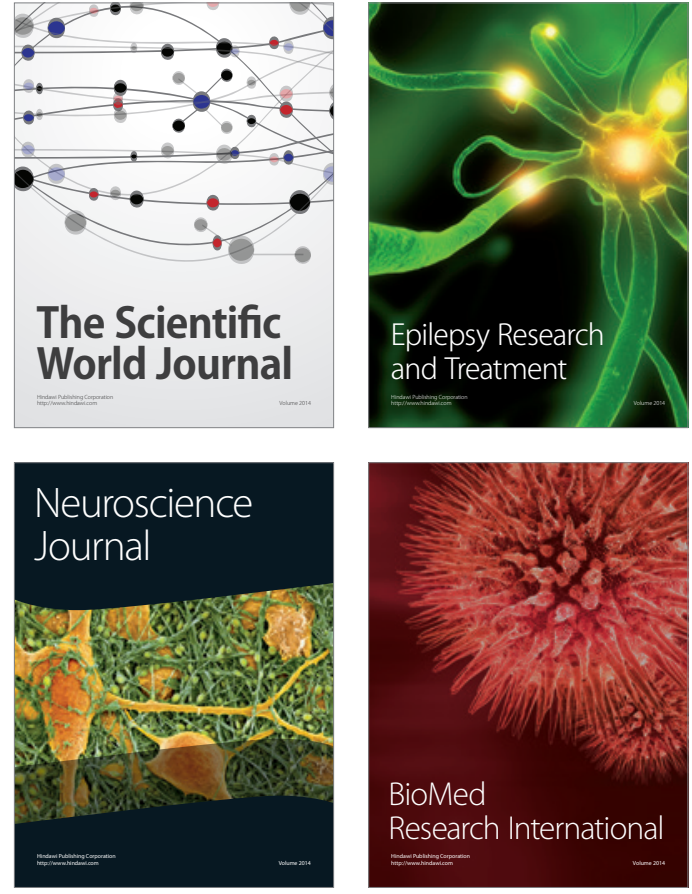

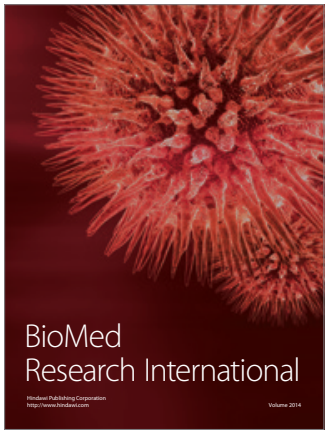

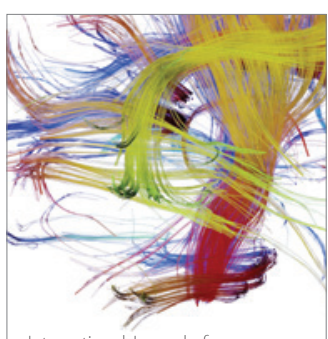

Brain Science

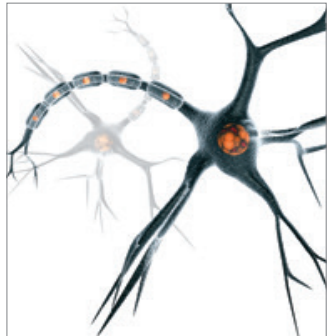

Neural Plasticity
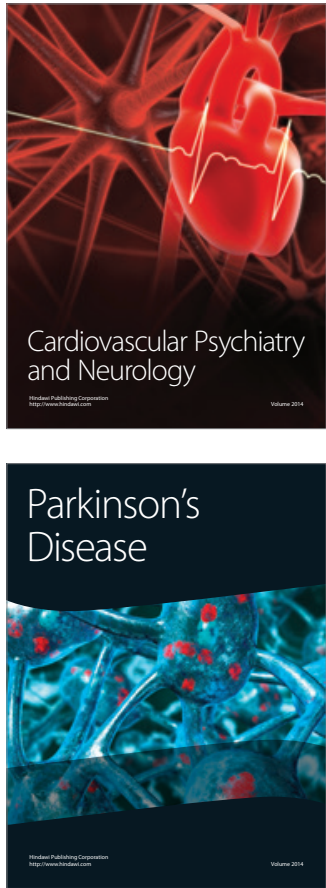